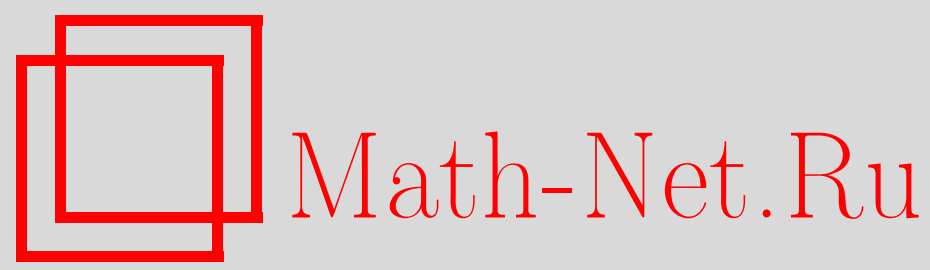

В. А. Колмыков, Отражения и трехмерность, Матем. заметки, 2003, том 73, выпуск 6, 848-852

DOI: https://doi.org/10.4213/mzm232

Использование Общероссийского математического портала Math-Net.Ru подразумевает, что вы прочитали и согласны с пользовательским соглашением http://www.mathnet.ru/rus/agreement

Параметры загрузки:

IP: 18.209.158.208

26 апреля 2023 г., $17: 33: 37$

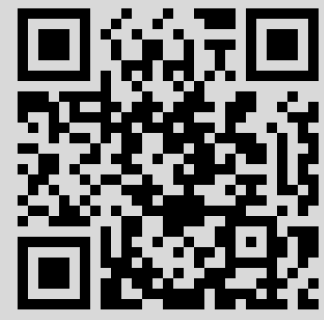




\section{ОТРАЖЕНИЯ И ТРЕХМЕРНОСТЬ}

\section{В. А. Колмыков}

Если размерность линейного пространства $\leqslant 3$, то характеристический многочлен преобразования Кокстера, ассоциированного с любой симметрической матрицей, инвариантен относительно естественного действия симметрической группы. Если размерность больше 3, то это утверждение неверно. Множество всех деревьев, имеющих в спектре соответствующего преобразования Кокстера минус единицу, трехмерно.

Библиограффия: 4 названия.

1. Отражения и преобразование Кокстера. Линейньй оператор $R: E \rightarrow E$ назьвается отражением, если линейное пространство $E$ можно представить в виде прямой суммы $L \oplus H$ так, что $L$ одномерно, сужение $R$ на $H$ есть тождественное отображение, а сужение $R$ на $L$ есть умножение на -1 . Говорят, что $R$ - отражение относительно гиперплоскости $H$ вдоль прямой $L$. В [1] введен оператор, являющийся суперпозицией всех отражений относительно специального набора гиперплоскостей. Такой оператор стали называть преобразованием Кокстера, но из нужд теории, к которой его применяли, это понятие слегка обобщали. Одно из современных определений таково. Пусть $A$ - вещественная $(n \times n)$-матрица. Положим $R_{i}=I-A_{(i)}$, где $A_{(i)}-$ матрица, получающаяся из $A$ занулением всех элементов, кроме тех, которые стоят в

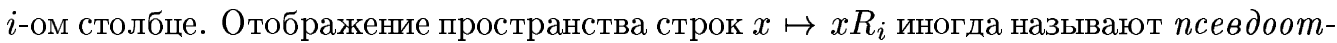
ражением ${ }^{1}$. Положим $C(A)=R_{1} R_{2} \cdots R_{n}$. Преобразованием Кокстера, связанным с матрицей $A$, назьвается преобразование пространства строк $x \mapsto x C(A)$.

2. Симметричность и независимость. Квадратную матрицу назовем независимой, если при любой перестановке строк и такой же перестановке столбцов характеристический многочлен соответствующего преобразования Кокстера не изменится.

Размерность 3 часто является естественным рубежом, преодолевая который, утверждение теряет истинность. Так происходит и с утверждением о независимости симметрических матриц.

Теорема 1. Следующие утверждения равносильны:

(1) любая вещественная симметрическая $(n \times n)$-матрича независима;

(2) число п меньше или равно 3.

\footnotetext{
${ }^{1}$ Оно будет отражением, если и только если $a_{i i}=2$.
} 
ДокАЗАТЕЛЬСтво. Для характеристического многочлена матрищы $C(A)$ существует красивая формула [2, гл. V, 6, упр. 3$]$ :

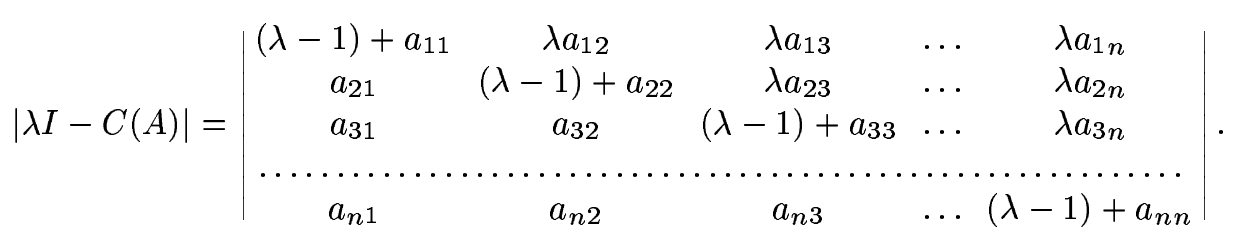

Докажем $(1) \Rightarrow(2)$. При $n=4$ утверждение (1) неверно: для

$$
A=\left(\begin{array}{cccc}
2 & -1 & 0 & -1 \\
-1 & 2 & -1 & 0 \\
0 & -1 & 2 & -1 \\
-1 & 0 & -1 & 2
\end{array}\right) \quad \text { и } A^{\prime}=\left(\begin{array}{cccc}
2 & -1 & -1 & 0 \\
-1 & 2 & 0 & -1 \\
-1 & 0 & 2 & -1 \\
0 & -1 & -1 & 2
\end{array}\right)
$$

характеристические многочлены соответствующих преобразований Кокстера равны $\lambda^{4}-\lambda^{3}-\lambda+1$ и $\lambda^{4}-2 \lambda^{2}+1$.

При $n \geqslant 5$ утверждение (1) неверно: достаточно рассмотреть матрицы

$$
\left(\begin{array}{ll}
O & O \\
O & A
\end{array}\right) \text { и }\left(\begin{array}{ll}
O & O \\
O & A^{\prime}
\end{array}\right) \text {, }
$$

где $A$ и $A^{\prime}$ - вышерассмотренные матрищы.

Докажем $(2) \Rightarrow(1)$. Пусть $A$ - произвольная комплексная $(3 \times 3)$-матрища. Тогда

$$
\begin{aligned}
\mid \lambda I & -C(A) \mid=(\lambda-1)^{3}+(\lambda-1)^{2}\left(a_{11}+a_{22}+a_{33}\right)+(\lambda-1)\left(a_{11} a_{22}+a_{22} a_{33}+a_{11} a_{33}\right) \\
& -\lambda(\lambda-1)\left(a_{13} a_{31}+a_{12} a_{21}+a_{23} a_{32}\right)-\lambda\left(a_{11} a_{23} a_{32}+a_{22} a_{13} a_{31}+a_{33} a_{12} a_{21}\right) \\
& +\left(a_{12} a_{23} a_{31} \lambda^{2}+a_{13} a_{32} a_{21} \lambda\right)+a_{11} a_{22} a_{33} .
\end{aligned}
$$

Очевидно, что все слагаемые, кроме $\left(a_{12} a_{23} a_{31} \lambda^{2}+a_{13} a_{32} a_{21} \lambda\right)$ инвариантны относительно перестановки строк с той же перестановкой столбцов. В случае же симметричности матрицы $A$, и это слагаемое инвариантно относительно указанного действия. Теорема 1 доказана.

3. Преобразования Кокстера, деревья и минус единица. В п. 1 мы дали определение преобразования Кокстера как оператора, действующего в пространстве $\mathbb{R}^{n}$. Похожим образом ${ }^{2}$ можно дать определение преобразования Кокстера произвольного вещественного линейного конечномерного пространства $E$.

В теории представлений [3] используется важньй частный случай такого определения, когда преобразование Кокстера строится по некоторому графоу ${ }^{3} G$. Пусть $V(G)-$ множество всех вершин графа $G$. Всякая вешественная функция, определенная на $V(G)$,

\footnotetext{
${ }^{2}$ Схожесть состоит в том, что введение в $E$ специального базиса и отождествление этого пространства с $\mathbb{R}^{n}$ индуцирует отождествление преобразования Кокстера с тем, которое было определено первым способом.

${ }^{3}$ В настоящей статье под словом "граф" понимается конечный граф без петель и кратных ребер. Иньми словами, графф - это пара $(V, U)$, где $V$ - конечное множество, а $U$ - некоторое множество, состоящее из двухэлементньх подмножеств $V$.
} 
назьвается взвешиванием. Взвешивания естественно образуют $|V(G)|$-мерное пространство. С каждой вершиной $u$ связано преобразование $\sigma_{u}$ этого пространства определяемое следуюшим образом: $\sigma_{u}$ не меняет весов вершин, отличных от $u$, а вес вершины $u$ заменяет на противоположньй плюс сумма весов всех вершин, смежных с $u$. Операторы $\sigma_{u}$ являются отражениями. Преобразованием Кокстера назьвается суперпозиция всех отражений $\sigma_{u}$, взятых по одному (порядок произвольньй).

Итак, с каждым графом связано, вообще говоря, несколько преобразований Кокстера. Оказывается, если граф является деревом, то все эти преобразования Кокстера сопряжены (см. [3]). В этом случае все они имеют одинаковый спектр, которьй назьвается спектром дерева.

В [4] была показана важная роль минус единицы по отношению к спектрам деревьев. Сейчас мы покажем, что множество $\mathscr{T}_{-}$всех деревьев, содержащих минус единицу в спектре, является в некотором смысле трехмерным.

4. Свободная склеечная размерность. Вложением $f: T_{1} \rightarrow T_{2}$ деревъев назьвается инъективное отображение множеств их вершин $f: V\left(T_{1}\right) \rightarrow V\left(T_{2}\right)$ такое, что если вершины $u$ и $v$ соединены ребром в $T_{1}$, то $f(u)$ и $f(v)$ соединены ребром в $T_{2}$.

Будем говорить, что дерево $T$ склеено из деревьев $T_{1}$ и $T_{2}$, если существуют вложения $f_{i}: T_{i} \rightarrow T$ такие, что $f\left(V\left(T_{1}\right)\right) \cup f_{2}\left(V\left(T_{2}\right)\right)=V(T)$.

Пусть $\mathscr{H}$ - некотороемножество деревьев. Бинарную частичную многозначную операцию $*$ на множестве $\mathscr{H}$ (т.е. отображение $\mathscr{H}^{2} \rightarrow 2^{\mathscr{H}}$ ) назовем склейкой, если для любых $T_{1}, T_{2} \in \mathscr{H}$ любое $T \in T_{1} * T_{2}$ (если, конечно, множество $T_{1} * T_{2}$ непусто) склеено из $T_{1}$ и $T_{2}$. Из определения склейки следует, что всякое дерево из $T_{1} * T_{2}$ имеет число вершин меньшее или равное сумме чисел вершин $T_{1}$ и $T_{2}$. В частности, множество $T_{1} * T_{2}$ конечно.

Если $T$ склеено из $T_{1}$ и $T_{2}$, а $f_{i}: T_{i} \rightarrow T, i=1,2,-$ соответствующие вложения, то можно определить граф $S$, имеющий $f_{1}\left(V\left(T_{1}\right)\right) \cap f_{2}\left(V\left(T_{2}\right)\right)$ множеством вершин, причем вершины $u$ и $v$ соединены ребром в графе $S$, если и только если они соединены ребром в $T$ (если множество $f_{1}\left(V\left(T_{1}\right)\right) \cap f_{2}\left(V\left(T_{2}\right)\right)$ пусто, то $S$ - пустой граф, обозначаемьй Emp; очевидно, что если $S \neq \mathrm{Emp}$, то $S$ - дерево). В деревьях $T_{1}$ и $T_{2}$ существуют соответственно подграфы $S_{1}$ и $S_{2}$, изоморфные $S$ и такие, что $f_{1}\left(V\left(S_{1}\right)\right)=V(S)=f_{2}\left(V\left(S_{2}\right)\right)$. Будем говорить, что $T$ склеено из $T_{1}$ и $T_{2}$ посредством $S$-отождествления.

Пусть $\mathscr{H}$ - некоторое множество деревьев, а * - некоторая склейка, определенная на нем. Склейку * назовем свободной, если для любой пары $\left(T_{1}, T_{2}\right)$ деревьев из множества $\mathscr{H}$ выполнено условие: если $T_{1} * T_{2}$ непусто, то существует граф $S_{*}\left(T_{1}, T_{2}\right)$ такой, что $T_{1} * T_{2}$ состоит из всех деревьев, которые можно получить из $T_{1}$ и $T_{2}$ посредством $S_{*}\left(T_{1}, T_{2}\right)$-отождествления. Таким образом, с каждой свободной склейкой $*$ связано частичное отображение $S_{*}: \mathscr{H}^{2} \rightarrow \mathscr{T} \cup\{\operatorname{Emp}\}$.

Пусть $\mathscr{M}$ - множество, $\mathscr{P}$ - его подмножество, $\mathscr{R}$ - набор операций (возможно, частичных и многозначных), позволяющих из элементов $\mathscr{P}$ строить какие-то элементы $\mathscr{M}$, а из полученных - другие и т.д. Если в результате такого конструирования из $\mathscr{P}$ посредством $\mathscr{R}$ получается все $\mathscr{M}$, то пару $(\mathscr{P}, \mathscr{R})$ назовем конструктором множества $\mathscr{M}$; обозначается $\mathscr{M}=\langle\mathscr{P} \mid \mathscr{R}\rangle$.

Конструктор $\mathscr{K}=\langle\mathscr{P} \mid *\rangle$ назовем свободным склеечны.м, если $*-$ свободная склейка. Мощностью саrd $\mathscr{K}$ такого конструктора назовем мощность множества $\mathscr{P}$.

Пусть $\mathscr{H}$ - некоторое множество деревьев. Свободной склеечной размерностью $\mathscr{H}$ назовем inf $\{$ card $\mathscr{K}\}$, где $\mathscr{K}$ пробегает множество всех свободных склеечных конструкторов множества $\mathscr{H}$. 
Теорема. Свободная склеечная размерность $\mathscr{T}_{-}$равна 3.

ДокАЗАТЕЛЬСТво. На множестве всех деревьев определим бинарную многозначную операцию $\ominus$ следующим образом. Если $T_{1}$ и $T_{2}-$ деревья, то множество $T_{1} \ominus T_{2}$ состоит из всевозможны $x$ результатов соединений этих деревьев ребром (в $T_{1}$ и $T_{2}$ произвольным образом фиксируется по одной вершине, затем отмеченные вершины соединяются ребром). Все деревья из $T_{1} \ominus T_{2}$ и только они получаются из $T_{1}$ и $T_{2}$ посредством Emp-отождествления. Операция $\ominus$ коммутативна. Она является свободной склейкой.

Звездой $Z_{n}$ называется $(n+1)$-вершинное дерево, в котором есть вершина степени $n$. Через $\mathscr{T}_{+}$обозначим множество всех деревьев, не имеющих в спектре минус единицу. В [4] было доказано, что $\mathscr{T}_{+}=\left\langle Z_{1} \mid \ominus\right\rangle$.

1) Предположим, что свободная склеечная размерность $\mathscr{T}_{-}$равна 1 и $\mathscr{T}_{-}=\langle X \mid *\rangle$. Тогда $X=Z_{0}$, так как $Z_{0} \in \mathscr{T}_{-}$и $Z_{0}$ нельзя склеить из других деревьев, кроме $Z_{0}$. Если $S_{*}\left(Z_{0}, Z_{0}\right)=\mathrm{Emp}$, то $Z_{0} * Z_{0}=\left\{Z_{1}\right\}$, но $Z_{1} \in \mathscr{T}_{+}$. Если $S_{*}\left(Z_{0}, Z_{0}\right)$ не определено или равно $Z_{0}$, то $\mathscr{T}_{-}=\left\langle Z_{0} \mid *\right\rangle$ - одноэлементное множество. Противоречие.

2) Предположим, что свободная склеечная размерность $\mathscr{T}_{-}$равна 2 и $\mathscr{T}_{-}=\langle X, Y \mid *\rangle$. Так как $Z_{0}$ нельзя склеить из других деревьев, кроме $Z_{0}$, то одно из деревьев $X$ или $Y$ (для определенности, скажем, $X$ ) равно $Z_{0}$.

Если $S_{*}\left(Z_{0}, Z_{0}\right)=\mathrm{Emp}$, то $Z_{0} * Z_{0}=\left\{Z_{1}\right\}$, но $Z_{1} \notin \mathscr{T}_{-}$. Если $S_{*}\left(Z_{0}, Z_{0}\right)$ неопределено или равно $Z_{0}$, то $Z_{0} * Z_{0} \subseteq\left\{Z_{0}\right\}$, поэтому $Y \notin\left\langle Z_{0} \mid *\right\rangle$. Так как $Z_{2}$ нельзя склеить из элементов множества $\mathscr{T}_{-} \backslash\left\{Z_{0}\right\}$, то $Y=Z_{2}$.

Заметим, что $S_{*}\left(Z_{0}, Z_{0}\right) \neq \operatorname{Emp}$ (иначе бы $\left.Z_{0} * Z_{0} \ni Z_{1} \notin \mathscr{T}_{-}\right), S_{*}\left(Z_{0}, Z_{2}\right) \neq \mathrm{Emp}$ (иначе $Z_{0} * Z_{2}$ содержало бы 4-вершинную цепь $A_{4}$, но $A_{4} \in\left\langle Z_{1} \mid \ominus\right\rangle=\mathscr{T}_{+}$), аналогично $S_{*}\left(Z_{2}, Z_{0}\right) \neq$ Emp. Поэтому $S_{*}\left(Z_{0}, Z_{0}\right)=Z_{0}$ или неопределено, $S_{*}\left(Z_{0}, Z_{2}\right)=Z_{0}$ или неопределено, $S_{*}\left(Z_{2}, Z_{0}\right)=Z_{0}$ или неопределено. В любом из восьми возможных случаев можно утверждать, что

$$
Z_{0} * Z_{0} \subseteq\left\{Z_{0}\right\}, \quad Z_{0} * Z_{2} \subseteq\left\{Z_{2}\right\} \supseteq Z_{2} * Z_{0}
$$

Если $S_{*}\left(Z_{2}, Z_{2}\right)$ неопределено, то $Z_{2} * Z_{2}=\varnothing$, а $\mathscr{T}_{-}=\left\langle Z_{0}, Z_{2} \mid *\right\rangle$ - двухэлементное множество. Противоречие.

Если $S_{*}\left(Z_{2}, Z_{2}\right)=\mathrm{Emp}$, то $Z_{2} * Z_{2}$ (а вместе с ним и $\mathscr{T}_{-}$) содержит 6-вершинную цепь $A_{6}$, но $A_{6} \in\left\langle Z_{1} \mid \ominus\right\rangle=\mathscr{T}_{+}$. Противоречие.

Если $S_{*}\left(Z_{2}, Z_{2}\right)=Z_{0}$, то все деревья из $Z_{2} * Z_{2}$ имеют пять вершин. Из 5-вершинного дерева и дерева из множества $\left\{Z_{0}, Z_{2}\right\}$ не склеить $Z_{3}$, поэтому $Z_{3} \notin\left\langle Z_{0}, Z_{2} \mid *\right\rangle=\mathscr{T}$. Противоречие.

Если $S_{*}\left(Z_{2}, Z_{2}\right)=Z_{1}$, то $Z_{2} * Z_{2}$ (а вместе с ним и $\mathscr{T}_{-}$) содержит 4-вершинную цепь $A_{4}$, но $A_{4} \in\left\langle Z_{1} \mid \ominus\right\rangle=\mathscr{T}_{+}$. Противоречие.

Если $S_{*}\left(Z_{2}, Z_{2}\right)=Z_{2}$, то $Z_{2} * Z_{2}=\left\{Z_{2}\right\}$, отсюда $\mathscr{T}_{-}=\left\langle Z_{0}, Z_{2} \mid *\right\rangle$-двухэлементное множество. Противоречие.

3) На множестве всех деревьев определим бинарную многозначную операцию $\oplus$ следующим образом. Если $T_{1}$ и $T_{2}-$ деревья, то множество $T_{1} \oplus T_{2}$ состоит из всевозможных деревьев, получаюшихся из $T_{1}$ и $T_{2}$ посредством $Z_{0}$-отождествления. Операция $\oplus$ коммутативна. Она является свободной склейкой.

В [4] было доказано, что $\mathscr{T}_{-}=\left\langle Z_{0}, Z_{2}, Z_{3} \mid \oplus\right\rangle$, поэтому свободная склеечная размерность $\mathscr{T}_{-}$меньше или равна трем. Теорема 2 доказана. 


\section{СПИСОК ЦИТИРОВАННОЙ ЛИТЕРАТУРЫ}

[1] Coxeter H. S. M. The product of generators of finite group generated by reflections // Duke Math. J. 1951. № 18. P. 765-782.

[2] Бурбаки Н. Группы и алгебры Ли. Группы Кокстера и системы Титса. Группы, порожденные отражениями. Системы корней: Пер. с франц. М.: Мир, 1972.

[3] Бернштейн И. Н., Гельфанд И. М., Пономарев В. А. Функторы Кокстера и теорема Габриеля // УМН. 1973. Т. 28. № 2. С. 19-33.

[4] Колмыков В.А. Преобразование Кокстера и число “-1" // Матем. сб. 2000. Т. 191. № 10. C. $51-56$.

Воронежский государственный университет 